\title{
Trabecular Juvenile Ossifying Fibroma of the Craniofacial Skeleton: Etiopathogenesis and a Case Report of the Rare Entity
}

\author{
${ }^{1}$ Rahul Kadam, ${ }^{2}$ Shilpa Patel, ${ }^{3}$ Jigna Pathak, ${ }^{4}$ Niharika Swain, ${ }^{5}$ Shwetha Kumar
}

\begin{abstract}
The term, fibro-osseous lesions, is used for a group of pathological disturbances encompassing developmental, reactive or dysplastic lesions and neoplasms characterized by replacement of normal bone architecture by tissue composed of collagen fibers and fibroblasts containing various amount of calcified tissue. The groups of the fibro-osseous lesions are best considered as a spectrum of processes arising from cells in the periodontal ligament. Juvenile ossifying fibroma (JOF) is a benign, but potentially aggressive, fibro-osseous tumor of the craniofacial bones. This uncommon neoplasm is distinguished from other fibro-osseous lesions primarily by its age of onset, clinical presentation, potential behavior and the high tendency to recur. Clinically presenting as an actively growing lesion. Histopathologically consists cell rich fibrous stroma containing bands of cellular osteoid without osteoblastic lining together with trabeculae of more typical woven bone. Pathogenesis of JOF may be related to mutations of HRPT2 gene which may arises due to haploinsufficiency of the HRPT2 gene.

Here, we reported a case of trabecular JOF (TJOF) which had variations in clinical, radiographic features and histopathological characteristics and it's etiopathogenesis in detail.
\end{abstract}

Keywords: Fibro-osseous lesions, Juvenile ossifying fibroma, Trabecular, Maxilla, Mandible.

How to cite this article: Kadam R, Patel S, Pathak J, Swain $\mathrm{N}$, Kumar S. Trabecular Juvenile Ossifying Fibroma of the Craniofacial Skeleton: Etiopathogenesis and a Case Report of the Rare Entity. J Contemp Dent 2014;4(1):51-55.

\section{Source of support: Nil}

Conflict of interest: None

\section{INTRODUCTION}

Juvenile ossifying fibroma (JOF) is a benign, but potentially aggressive, fibro-osseous tumor of the craniofacial bones. This uncommon bone forming neoplasm is distinguished from other fibro-osseous lesions primarily by its age of onset, clinical presentation, potential behavior and the high tendency

\footnotetext{
${ }^{1}$ Postgraduate Student, ${ }^{2}$ Professor and Head, ${ }^{3}$ Professor 4,5 Senior Lecturer

${ }^{1-5}$ Department of Oral Pathology, MGM Dental College and Hospital, Navi Mumbai, Maharashtra, India
}

Corresponding Author: Rahul Kadam, Postgraduate Student, Department of Oral Pathology, MGM Dental College and Hospital, Navi Mumbai, Maharashtra, India, Phone: 02227426604, e-mail: rahul33kadam@gmail.com to recur. ${ }^{1,2}$ The second edition of the WHO classification of odontogenic tumor defines JOF as 'an actively growing lesion consisting of a cell-rich fibrous stroma, containing bands of cellular osteoid without osteoblastic lining, together with trabeculae of more typical woven bone. Small foci of giant cells may also be present; the lesion is non-encapsulated but well-demarcated from surrounding bone'. ${ }^{3}$ This terminology is given to some rarely reported ossifying fibromas, mainly found in maxilla at an early age and in $79 \%$ of the patients are diagnosed before the age of 15 or younger which histologically showed a highly cellular but loose fibroblastic stroma containing strands of osteoid with entrapped osteoblast. ${ }^{4}$ The JOF occurs predominantly but not exclusively in children. It affects males and females equally without any gender predilection. ${ }^{5,6}$ The JOF is located mainly ( $85 \%$ ) in facial bones, in some cases $(12 \%)$ in calvarium and very rarely $(3 \%)$ extracranially. Ninety percent of the lesions located in the facial region. ${ }^{4}$ Mandibular lesions are seen in $10 \%$ of the cases. Clinically, the lesion seems to be expands in the facial bone leading to facial asymmetry. The clinical symptoms, such as pain, paraesthesia, malocclusion, sinusitis and proptosis depend upon the site of tumor involvement. Root displacement is common but root resorption is rare. The lesion can cause expansion as well as perforation. ${ }^{7}$

Radiographically, they present as a well-circumscribed radiolucencies that in some cases contain central radiopacities or they appear radiolucent, radiopaque or mixed radiolucent-radiopaque with a well-defined sclerotic border, lesions that are more aggressive may show cortical thinning and perforation. ${ }^{8}$ Being on the basis of different histopathological and clinical features in the WHO classification of odontogenic tumors 2005, juvenile ossifying fibroma (JOF) is further subdivided into juvenile psammomatoid ossifying fibroma (JPOF) and juvenile trabecular ossifying fibroma (JTOF). ${ }^{9,10}$

Here, we reported a case of trabecular type of JOF in 18-year-old male patient with a chief complaint of painless swelling in the lower right anterior region with its variation in clinical behavior, radiographic features and histopathological characteristics. 


\section{CASE REPORT}

An 18-year-old male patient reported to Department of Oral Pathology, MGM Dental College and Hospital, with the chief complaint of large painless swelling in the lower anterior region since 6 years. Patient was apparently normal 7 years back. He first noticed a small swelling which started growing progressively to attain the present size, there was no pain associated with the swelling. No aggravating or relieving factors were associated with the present growth. On general physical examination, the patient was conscious, cooperative, healthy, thin built and well-nourished. All vital signs were within the normal range.

Extraorally, a diffuse large swelling around $4 \times 3 \mathrm{~cm}$ in size was seen in mandibular lower right anterior region crossing the midline extending anteroposteriorly from left side of corner of mouth to $2 \mathrm{~cm}$ away from right side of angle of the mandible (Fig. 1). On palpation, the swelling was non-tender, non-compressible, non-fluctuant and without the ulceration of overlying mucosa. Due to swelling, there was displacement of buccal and lingual cortical plate. Intraorally, a solitary, well-defined swelling obliterating the vestibule and extending from mandibular lower right anterior region crossing the midline. On palpation, the consistency of the swelling was firm to hard and nontender, causing expansion of the buccal cortical plates (Fig. 2). Hematological findings were unremarkable. A radiological investigation includes orthopantomogram (OPG) and computed tomography (CT) scan. Lateral cephalogram showed an expansile growth in lower right anterior region with mixed radiolucent and radiopaque pattern extending from lower left anterior region to lower right posterior region involving and pushing the lower border of mandible inferiorly. There was diffuse

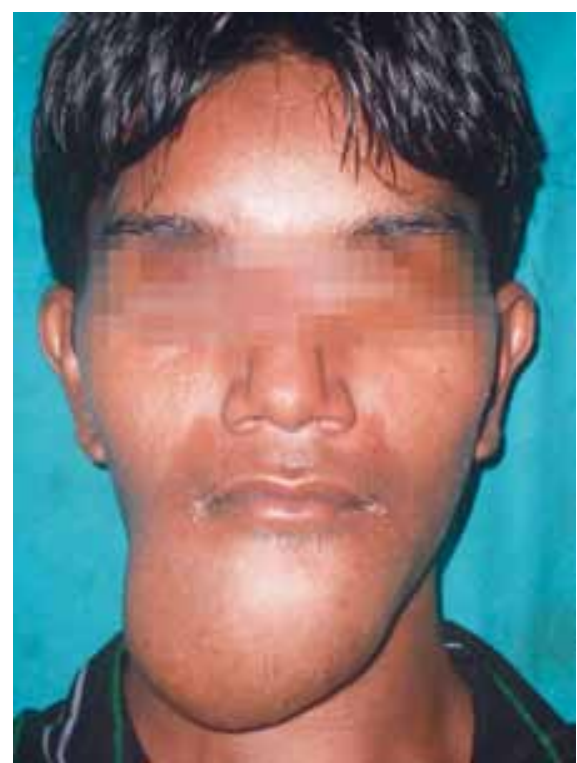

Fig. 1: Extraoral appearance of swelling radiopacities with ill-defined borders, but the overall lesion showed mixed pattern (Fig. 3). Considering the clinical features and radiological findings, a provisional diagnosis of ameloblastoma, CEOT and ossifying fibroma was made. After surgical exposure, an incisional biopsy was performed from the lesional tissues in mandibular anterior region, which was sent for histopathological analysis.

H\&E stained soft tissue section shows strands of highly cellular osteoid with plump and irregular osteocyte in hypercellular fibrous connective tissue stroma. Areas of pseudocystic degeneration and hemorrhage are seen (Figs 4 and 5). The overall histopathological features were suggestive of JTOF.

\section{DISCUSSION}

Juvenile ossifying fibroma is an uncommon benign osteogenic neoplasm, which comprises $2 \%$ of oral tumor in

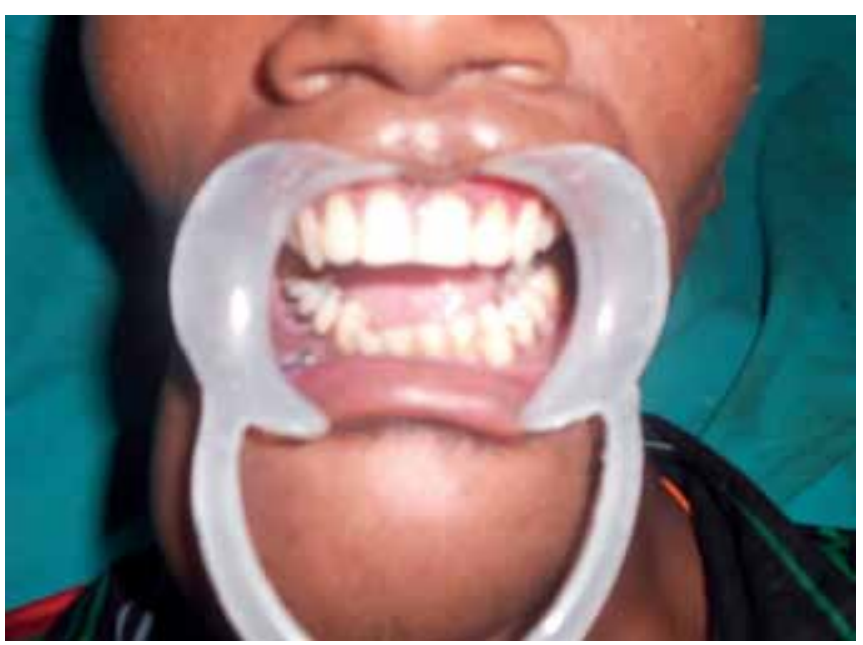

Fig. 2: Intraoral view shows a solitary, well-defined swelling obliterating the vestibule and extending from mandibular lower right anterior region crossing the midline

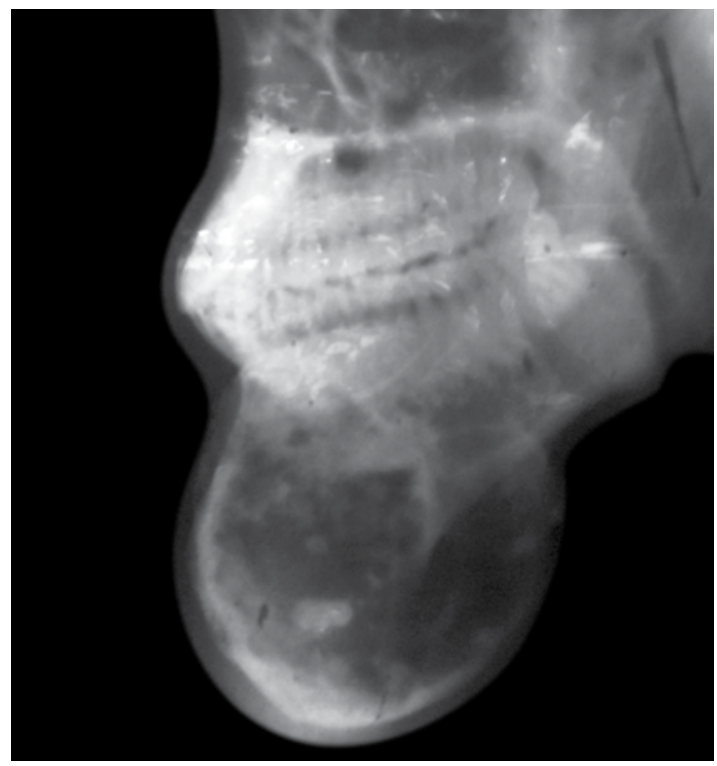

Fig. 3: An expansile growth in lower right anterior region with mixed radiolucent and radiopaque pattern 
children. Some cases are part of a syndrome including primary hyperparathyroidism (caused by adenoma or carcinoma of the parathyroid) and renal lesions, the so-called 'hyperparathyroidism-jaw tumor syndrome' (HPT-JT); this diagnosis can be important, because of the possible involvement of other family members having a risk of malignancy. ${ }^{11}$ Most common occurance of JOF is seen within facial bones in $85 \%$ of the patients. These lesions are generally more defined, but may displace the teeth and invade the adjacent bone. Extracranially, clavicle is involved in $12 \%$ of the cases. Few cases especially in children (below 15 years) showed rapid growth and have a tendency to recur. ${ }^{12-15}$ Gender predilection has been a matter of controversy; El-Mofty reported a male predilection, some authors claiming no predilection for either sex, whereas Johnson et al found a higher incidence in females. ${ }^{16}$

Johnson et al hypothesized that JOF originates from overproduction of the myxofibrous cellular stroma normally involved in the growth of the septa in the paranasal sinuses

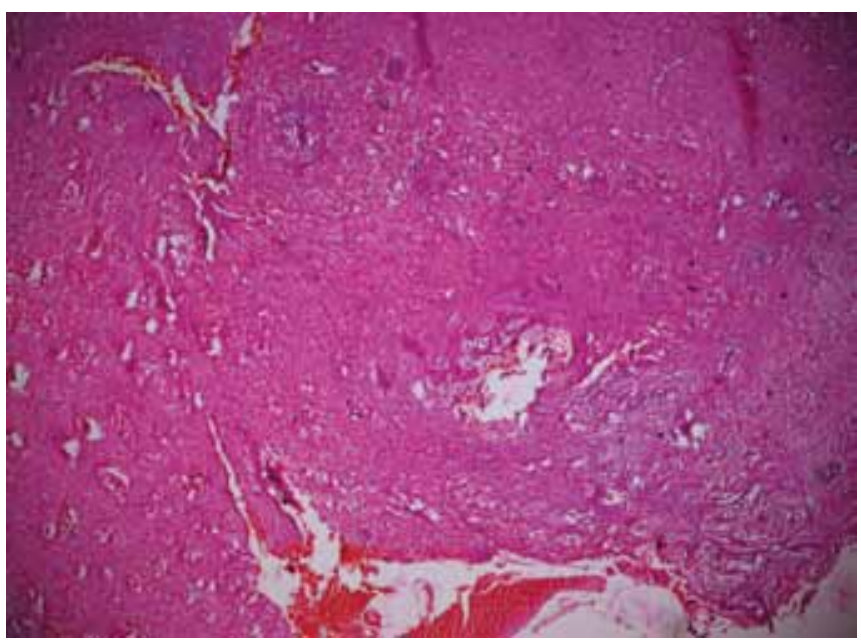

Fig. 4: H\&E $(\times 40)$ stained decalcified section shows strands of highly cellular osteoid with plump and irregular osteocyte in hypercellular fibrous connective tissue stroma

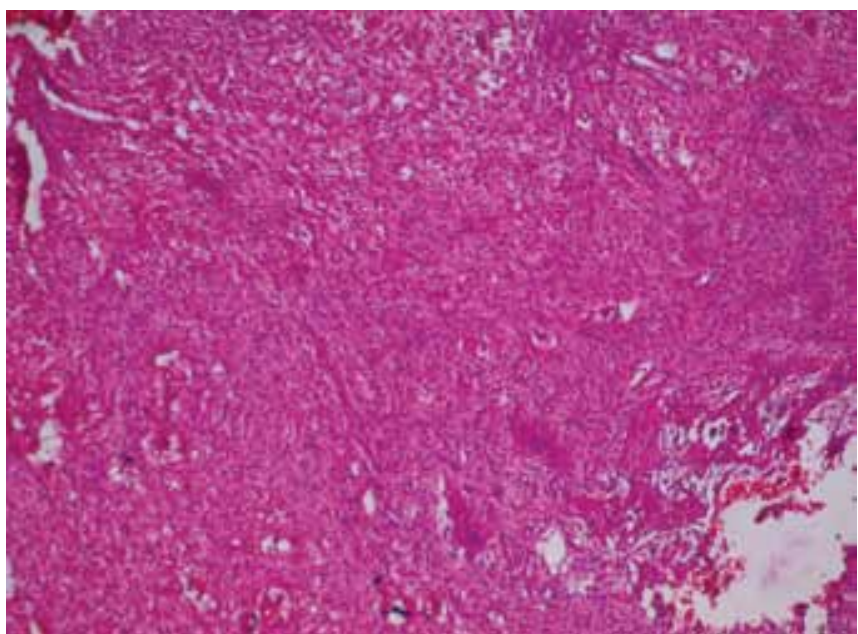

Fig. 5: H\&E (×100) stained decalcified section shows areas of pseudocystic degeneration and hemorrhage as they enlarge and pneumatize. These stromal cells secrete hyaline material that ossifies and connective tissue mucin that initiates the cystic areas. A recent study by Sawyer et al demonstrated the presence of nonrandom chromosome break points at Xq26 and 2q33 resulting in (X; 2) translocation. ${ }^{17}$

Pimenta et al identified mutations of HRPT2 gene in ossifying fibroma and suggest that ossifying fibroma may arise due to haploinsufficiency of the HRPT2 gene. But, such genetic alteration was absent in JOF indicating that it may be distinct clinicopathological entity. ${ }^{18}$

Some author considered that JOF may arise from the differentiation of mesenchymal cells of periodontal ligament (multipotent precursor cells) forming into fibrous tissue, cementum or osteoid. Because of its distinct histopathological features, it has been recognized as a separate entity among the fibro-osseous group of lesions. ${ }^{17}$ Trabecular variant of juvenile ossifying fibroma (JTOF) was first described by Reed and Hagy in 1965 and included under the designation of JOF. Makek (1983) was the first who published the largest series of tumor to date and reviewed 24 cases, 15 from the world literature and nine of his own and called the tumor as 'trabecular desmo-osteoblastoma'. ${ }^{18}$ He showed a slight male predilection (1.3:1) with maxilla being more commonly affected. Slootweg et al reported 10 cases showing a mean age of 11.8 years, again showing a male dominance, with $60 \%$ of the cases occurring in the maxilla. ${ }^{19}$ Samir El-Mofty in 2002 has mentioned patient age ranged from 3 to 23 years. Olga L Bohn et al (2011) have found JTOF occurs in young patients between 2 and 33 years. The tumor expands the affected bone, leading to facial asymmetry. ${ }^{20}$ Pain and paresthesia are rarely manifested. In the maxilla, the tumor may lead to nasal obstruction, epistaxis and eye displacement. The duration of the lesion is usually a few months to years. The present case was male patients and age of occurrence was 2 nd decade (18 years) but the location of lesion was different. In the present case, the location was in mandible which was since 6 years and nontender, nonaggressive in nature without displacement of teeth while. JTOF is characterized by a progressive and sometimes rapid aggressive growth but not all cases are characterized by a fast growth rate. Slow growth has also been reported in a few cases. ${ }^{21}$

Radiographic pattern depends upon the stage of development and amount of mineralized matrix present. JTOF is expansive, well-defined and unilocular or multilocular with cortical thinning and perforation. The tumor mass may show radiolucency with variable calcification induced radiopacity and occasionally producing a 'ground-glass' appearance. Increase in radio density may be observed over time. ${ }^{19}$ Root resorption and displacement of involved teeth may also observe. In the mandible, the angle and ramus 
is more commonly the site of involvement. ${ }^{22}$ Maxillary tumors tend to extend into and obliterate the maxillary sinus. Present both cases showed mixed radiolucent and radiopaque pattern giving a ground glass appearance. Histopathological examination of the TJOF shows a welldefined but noncapsulated lesion that infiltrates surrounding bone with reactive new bone formation at the periphery. ${ }^{21}$ The tumors had a characteristic loose structure with cell rich stroma composed of fibroblastic spindle cells that produced little collagen, as seen with the present two cases. Immature cellular osteoid formed anastomosing trabeculae in a pattern that resembles 'paintbrush strokes'. ${ }^{23}$ The cellular osteoid was not always clearly distinguished from the fibroblastic stroma. Calcification progressed into the osteoid strands, producing woven bone trabeculae that were rimmed with osteoblasts and incorporated osteocytes, as seen with the present case. Aggregates of osteoclastic giant cells (focal collections of multinucleated giant cells are commonly seen) in this case which were invariably present and seen in association with the bone trabeculae and in separate foci in the fibrous stroma, usually at sites of hemorrhage. ${ }^{24}$ Presence of proliferating cellular connective tissue with plump cells is suggestive of the aggressive nature of this neoplasm, as seen with the present case. The overall histopathological features were suggestive of JTOF. Fibro-osseous lesions present a diagnostic challenge due to overlapping clinical and histopathological findings. A differential diagnosis of fibrous dysplasia, malignant bony tumors, aneurysmal bone cyst, central giant cell granuloma, osteogenic sarcoma, osteoblastoma, calcifying odontogenic cyst, adenomatoid odontogenic tumor and primordial cysts (keratocyst) should be considered. JTOF is diagnosed by its clinical features, aggressive behavior and exclusive histopathological features. ${ }^{25,26}$

Surgical excision is the recommended treatment for JTOF. Because of infiltrating borders, the tumor cannot be shelled out but should be conservatively excised completely. Recurrences are seen in 30 to $50 \%$ of cases mostly due to incomplete excision resulting from the infiltrative nature of tumor border. In some cases, recurrence may occur more than once so more than one excision may be required to achieve complete cure. ${ }^{6-9}$ Radiotherapy has been proven ineffective and contraindicated due to the increased incidence of malignant transformation ranging from 0.4 to $0 \%$. Despite the aggressive behavior, no metastasis has been reported. ${ }^{11,14}$

\section{CONCLUSION}

Juvenile trabecular ossifying fibroma is a rare fibroosseous neoplasm found in the young age group, which is considered potentially aggressive than the conventional form which spreads quickly and has more chances of recurrence therefore early detection and complete surgical excision of these lesions followed by long-term follow-up bears importance in clinical management of these lesions.

\section{REFERENCES}

1. El-Mofty S. Psammomatoid and trabecular juvenile ossifying fibroma of the craniofacial skeleton: two distinct clinicopathologic entities. Oral Surg Oral Med Oral Pathol Oral Radiol Endod 2002;93:296-304.

2. Walter JM, Terry BC, Small EW, Matteson SR, Howell RM. Aggressive ossifying fibroma of the maxilla: review of the literature and report of case. J Oral Surg 1979;37:276-286.

3. Pindborg JJ, Hjorting-Hansen E. Juvenile ossifying fibroma. Atlas of diseases of the jaws. Copenhagen. Munksgaard 1974;68-70.

4. El-Mofty SK, Kyriakos M. Soft tissue and bone lesions. In: Gnepp DG, editor. Diagnostic surgical pathology of the head and neck. Philadelphia. WB Saunders 2001;505-604.

5. Makek MS. So-called fibro-osseous lesions of tumorous origin. Biology confronts terminology. J Craniomaxillofac Surg 1987; 15:154-167.

6. Makek M. Clinical pathology of fibro-osteocemental lesions of the cranio-facial skeleton and jaw bones. Basel (Switzerland), Karger 1983;128-227.

7. Slootweg PJ, Müller H. Juvenile ossifying fibroma. Report of four cases. J Craniomaxillofac Surg 1990;18:125-129.

8. Noffke CE. Juvenile ossifying fibroma of the mandible. An 8-year radiological follow-up. Dentomaxillofac Radiol 1998;27:363-366.

9. Halkias LE, Larsen PE, Allen CM, Steinberg MJ. Rapidly growing, expansile mass of the mandible in a 6-year-old boy. J Oral Maxillofac Surg 1998;56:866-871.

10. Williams HK, Mangham C, Speight PM. Juvenile ossifying fibroma. An analysis of eight cases and a comparison with other fibro-osseous lesions. J Oral Pathol Med 2000;29:13-18.

11. Bohn OL, et al. Trabecular and psammomatoid juvenile ossifying fibroma of the skull base mimicking psammomatoid meningioma. Head and Neck Pathol 2011;5:71-75.

12. Jundt G. Fibrous dysplasia. In: Barnes L, Eveson JW, Reichart P, Sidransky D, editors. Pathology and genetics of head and neck tumours. Lyon IARC 2005;321-322.

13. Voytek TM, Ro JY, Edeiken J, et al. Fibrous dysplasia and cemento-ossifying fibroma. A histologic spectrum. Am J Surg Pathol 1995;19:775-781.

14. Morikava FS, et al. Periapical cemento-osseous dysplasia: case report. RSBO 2012;9(1):102-107.

15. Dagistan $\mathrm{S}$, et al. Cemento-osseous dysplasias. Rev Clín Pesq Odontol 2007;3(1):43-49.

16. Benjellou L, et al. Florid osseous dysplasia: report of two cases and a review of the literature. Int J Odontostomat 2011; 5(3): 257-266

17. Mupparapu M, et al. Simultaneous presentation of focal cemento-osseous dysplasia and simple bone cyst of the mandible masquerading as a multilocular radiolucency. Dentomaxillo Facial Radiology 2005;34:39-43.

18. Kawai $\mathrm{T}$, et al. Cemento-osseous dysplasia of the jaws in 54 Japanese patients. Oral Surg Oral Med Oral Pathol Oral Radiol Endod 1999;87:107-114. 
19. Vegh T. Multiple cementomas (periapical cemental dysplasia): report of a case. Oral Surg Oral Med Oral Pathol 1976;42: 402-406.

20. Berkovitz BKB, Holland GR, Moxham BJ. Color atlas and textbook of oral anatomy, histology and embryology. 2nd ed. St Louis: Mosby-Year Book 1992;191-192.

21. Di Fiore PM, Cemento-osseous dysplasia in African-American men: a report of two clinical cases. J Tennessee Dent Assoc 2010; 90(4):24-28.

22. Bencharit S, Schardt-Sacco D, Zuniga JR, Minsley GE. Surgical and prosthodontic rehabilitation for a patient with aggressive florid cemento-osseous dysplasia: a clinical report. J Prosthet Dent 2003;90:220-224.

23. Dumas M, Ohanian H, Forest D. A case report of fluoride cemento-osseous dysplasia. J Dent Que 2000;37:97-101.

24. Melrose RJ. The clinico-pathologic spectrum of cemento-osseous dysplasia. Oral Maxillofac Clin Nor Am 1997;9:643-653.

25. Yazicioglu D, et al. Focal cemento-osseous dysplasia: a case report and literature review. Health 2010;941-944.

26. Radojica, et al. Focal cemento-osseous dysplasia in the maxilla mimicking periapical granuloma. Oral Surg Oral Med Oral Pathol Oral Radiol Endod 1999;88:87-89. 